This is a print version of the following published document:

C.Vázquez, A.Tapetado, H. Miguélez (2014). "Monitoring temperature on machining processes is enhanced using optical fibers" In SPIE Newsroom, 3 July, pp. 1-2. Available in http://www.dx.doi.org/10.1117/2.1201406.005510

(C) 2014 SPIE 


\section{Monitoring machining temperature using optical fibers}

\section{Carmen Vázquez, Alberto Tapetado, and Henar Miguélez}

An IR fiber-optic pyrometer measures temperatures above $250^{\circ} \mathrm{C}$ close to rotating components where other sensing techniques are unsuitable.

A strong trend in the aero-engine industry toward reducing engine weight and airline demands for performance improvements are driving efforts to reduce both fuel consumption and emissions. Engine designers are meeting these demands by introducing material that is ever more difficult to machine and generates more heat when machined. Temperature plays an important role in machined surface burns (resulting in surface roughness, imperfections, and residual stresses in the finished component) and higher tool wear rates, which shorten tool life. Tool wear is one of the limiting factors for manufacturing productivity levels. Measuring and controlling temperature in precise locations holds the key to extending tool life and component functional integrity.

Reported ways to measure cutting temperature in some machining processes include thermocouples and IR cameras. These techniques have been reviewed by Barrow and Komanduri among others. ${ }^{1,2}$ But in most applications, the rotating work piece and the cutting tool are very close and the temperature gradient so high that extremely local measurements are needed. In such cases, thermocouples as contact sensors interfere with the flow of heat, require sufficient time for temperature equilibrium to be reached, and cannot be easily located in limited space. IR thermography is non-intrusive, but the need to know the surface emissivity, the difficulty of installation with a free line of sight from the work piece to the sensor, and its unsuitability for very local measurements are significant disadvantages.

More recently, Müller and Beno, among others, have used an optical fiber to carry IR radiation from the work piece to a photodetector and used the power ratio of two sensors detecting signals at different wavelength ranges to avoid the influence of the temperature and material dependent emissivity. ${ }^{3,4}$ Optical fibers can be placed to measure localized temperature on machined surfaces and can be embedded in different sections. However,

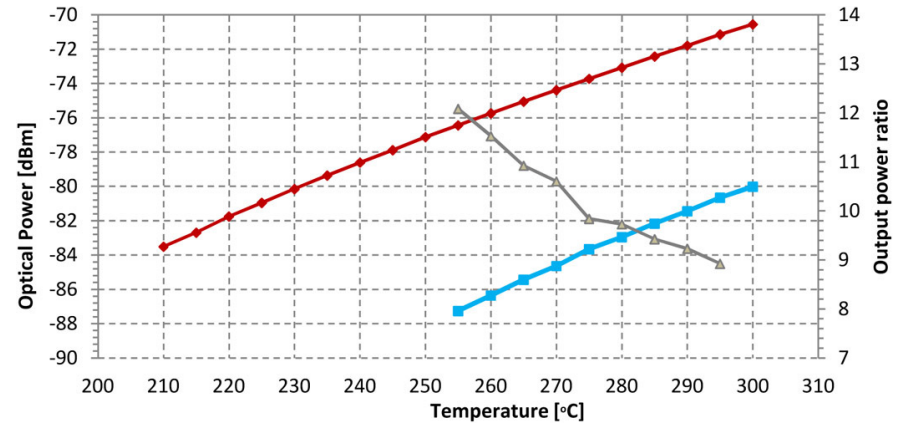

Figure 1. Sensor output power characterization versus temperature for (red) the upper wavelength range, (blue) the lower wavelength range, and (gray) the ratio between them.

this adds the expense of using two different detectors and reduces sensitivity and temperature range. Most of the reported measured temperatures are greater than $500^{\circ} \mathrm{C}$, and placing the sensor requires the cutting machine itself to be modified. To get around some of these problems, we selected wavelength ranges and fiber-optic components that are compatible with commercial off-the-shelf optoelectronics and wavelength division multiplexing optical communication networks. We placed the sensor in the cutting tool holder to avoid modifying the machine. ${ }^{5}$

The two-color pyrometer was calibrated using a black body and a heater and control thermal unit. The sensor was made of standard graded index silica fiber OM1 with 62.5/125 core and cladding diameters in microns, and the buffer coating was partially removed at the sensing end. A fiber-optic demultiplexer separated the signals at both wavelength ranges around 1300 and 1550nm. Figure 1 shows the optical power measurements. The lower wavelength range delimited the minimum temperature that the system can measure, and the power ratio was used to avoid the influence of emissivity variations versus calibration conditions. Remote control operation was performed using LabVIEW software. ${ }^{6}$

Figure 2 shows a photograph of the fiber optic embedded on the standard tool holder of a cutting tool (Sandvik TCMW 


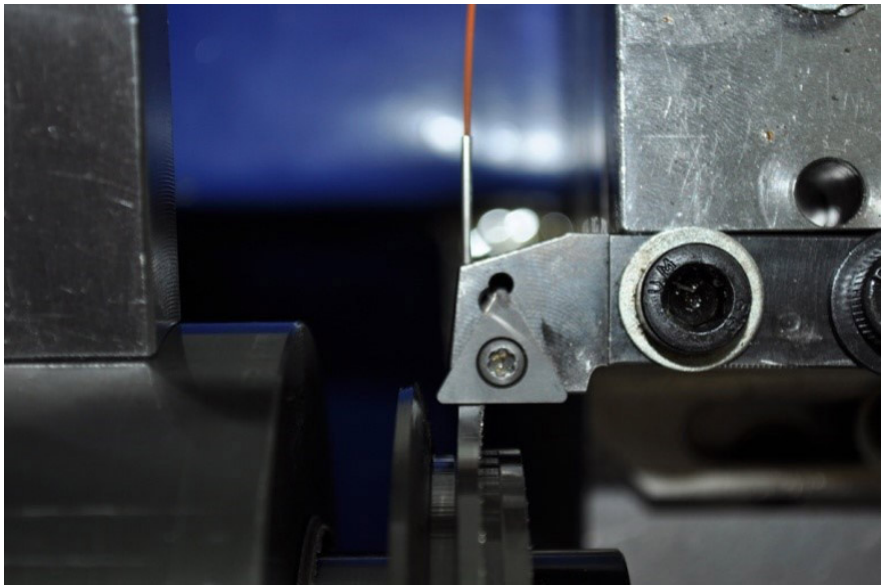

Figure 2. Optical fiber pyrometer placement during machining.

16 T3 08) and perpendicular to the cutting surface within a Pinacho CNC Smart 8165 machine. The machined material in the experiment is Inconel 718. We analyzed the correlation between temperature evolution and applied forces along the machining process and found they performed as expected. Cutting forces were measured with a tool dynamometer (Kistler 9257B) and a data acquisition system (Keithley 3100).

In summary, our fiber-optic pyrometer designs show promise for analyzing tool wear and component functional integrity after machining. So far our results have shown that we can easily measure temperatures for different materials from $250^{\circ} \mathrm{C}$ upward from IR radiation, after black body calibration of the system. The results are sufficiently clear to be useful in real machining processes for which we have also obtained preliminary results. Our next step will be to characterize the machining of new materials at different cutting speeds.

This work has been sponsored by the Spanish Ministry of Economy and Competitiveness under grant TEC2012-37983-C03-02.

\section{Author Information}

\section{Carmen Vázquez, Alberto Tapetado, and Henar Miguélez}

University of Carlos III of Madrid (UC3M)

Leganes, Spain

Carmen Vázquez is a professor at the Department of Electronic Technology and head of the Displays and Photonics Group at UC3M. Her research focus is on optical fiber sensors, integrated optics, and networks. She has more than 200 publications and has been a visiting scientist at the Massachusetts Institute of
Technology (MIT). She previously worked at the telecommunications companies TELECOM, Denmark, and Telefónica I+D, Spain.

Alberto Tapetado is a $\mathrm{PhD}$ candidate at UC3M. His research interests are optical fiber sensors and integrated optics for monitoring temperature and self-reference techniques. He has been a visiting student at Aston University, UK, and MIT and received a degree in industrial engineering from the University of CastileLa Mancha, Spain.

Henar Miguélez is a full professor in the Mechanical Engineering Department at UC3M, where she obtained her $\mathrm{PhD}$ and is also head of the Manufacturing Technologies and Components Design Group. Her research interests focus on machining tools and techniques, and modeling of machining processes and defects. She received a degree in aeronautical engineering from the Polytechnic University of Madrid, Spain.

\section{References}

1. G. Barrow, A review of experimental and theoretical techniques for assessing cutting temperatures, Ann. CIRP 22 (2), pp. 203-211, 1973.

2. R. Komanduri and Z. B. Hou, A review of the experimental techniques for the measurement of heat and temperatures generated in some manufacturing processes and tribology, Tribology Int'1 34, pp. 653-682, 2001.

3. B. Müller and U. Renz, Development of a fast fiber-optic two-color pyrometer for the temperature measurement of surfaces with varying emissivities, Rev. Sci. Inst. 72, pp. 3366-3374, 2001

4. T. Beno and U. Hulling, Measurement of cutting edge temperature in drilling, Proc. Conf. Manuf. Syst. CIRP 3, pp. 531-536, 2012.

5. A. Tapetado, C. Vázquez, X. Soldani, H. Miguélez, and D. S. Montero, Temperature sensor based on fiber optic pyrometer in material removal processes, Proc. SPIE 8421, p. 84212 V, 2012. doi: $10.1117 / 12.970276$

6. http://www.ni.com/labview/ Homepage of National Instruments LabVIEW system design software. Accessed 29 May 2014. 